\title{
G-CSF-producing Undifferentiated Pleomorphic Sarcoma Adjacent to the Ascending Colon and in the Right Iliopsoas Muscle: A Case Report and Review of the Literature
}

\author{
Shinsuke Kazama ${ }^{1}$, Tabu Gokita ${ }^{2}$, Michitoshi Takano ${ }^{1}$, Ayataka Ishikawa ${ }^{3}$, Yu Nishimura ${ }^{3}$, \\ Hiroaki Ishii ${ }^{1}$, Yusuke Nishizawa ${ }^{1}$, Yoji Nishimura ${ }^{1}$, \\ Yoshiyuki Kawashima ${ }^{1}$ and Hirohiko Sakamoto ${ }^{1}$
}

\begin{abstract}
:
Undifferentiated pleomorphic sarcoma (UPS) is a soft tissue sarcoma, occurring most commonly on the lower extremities. We herein report a rare case of primary UPS adjacent to the ascending colon and in the right iliopsoas muscle. Computed tomography of the abdomen revealed large masses, and the patient experienced a high-grade fever, leukocytosis, elevated serum C-reactive protein level, and hematopoietic activation on ${ }^{18} \mathrm{~F}$-fluorodeoxyglucose-positron emission tomography. This inflammatory reaction was caused by granulocyte colony-stimulating factor secreted by tumor cells. Surgical resection was performed, and the inflammatory reaction disappeared immediately. The patient received adjuvant chemotherapy and survived one year after the operation without evidence of recurrence.
\end{abstract}

Key words: undifferentiated pleomorphic sarcoma, inflammatory reaction, granulocyte colony-stimulating factor, ascending colon, iliopsoas muscle

(Intern Med 58: 2783-2789, 2019)

(DOI: 10.2169/internalmedicine.2762-19)

\section{Introduction}

In adults, malignant fibrous histiocytoma (MFH) is a soft tissue sarcoma, occurring most commonly on the lower extremities and in the abdominal cavity or retroperitoneum (1). MFH in the viscera, particularly with gastrointestinal involvement, is rare (2-22). In 2002, MFH was re-classified as undifferentiated pleomorphic sarcoma (UPS) by the World Health Organization (23). It is classified into three types: UPS, UPS with giant cells, and UPS with prominent inflammation.

Among these types, inflammatory UPS occurring in the gastrointestinal tract is extremely rare $(2,8,12,17,19,24)$. Inflammatory UPS has been reported to be correlated with the production of granulocyte colony-stimulating factor (GCSF) by tumor cells $(12,25)$.
We herein report a rare case of G-CSF-producing UPS adjacent to the ascending colon and in the right iliopsoas muscle. Although this inflammatory reaction caused by GCSF was noted preoperatively, it disappeared immediately after complete surgical resection of the tumors.

\section{Case Report}

A 50-year-old man was admitted to a local hospital because of abdominal pain and numbness in the right lower limb accompanied by a slight fever. Computed tomography (CT) of the abdomen revealed large well-defined enhanced heterogeneous masses: a $7.2 \times 6.0-\mathrm{cm}$ mass on the right side of the abdomen (Fig. 1A), which was adjacent to the ascending colon, and a $3.7 \times 3.6-\mathrm{cm}$ mass in the right iliopsoas muscle (Fig. 1B).

The patient was referred to our hospital with a $15-\mathrm{kg}$ loss

${ }^{1}$ Department of Gastroenterological Surgery, Saitama Cancer Center, Japan, ${ }^{2}$ Department of Orthopedic Surgery, Saitama Cancer Center, Japan and ${ }^{3}$ Department of Pathology, Saitama Cancer Center, Japan

Received: January 31, 2019; Accepted: April 1, 2019; Advance Publication by J-STAGE: June 27, 2019

Correspondence to Dr. Shinsuke Kazama, kaz-tky@umin.ac.jp 


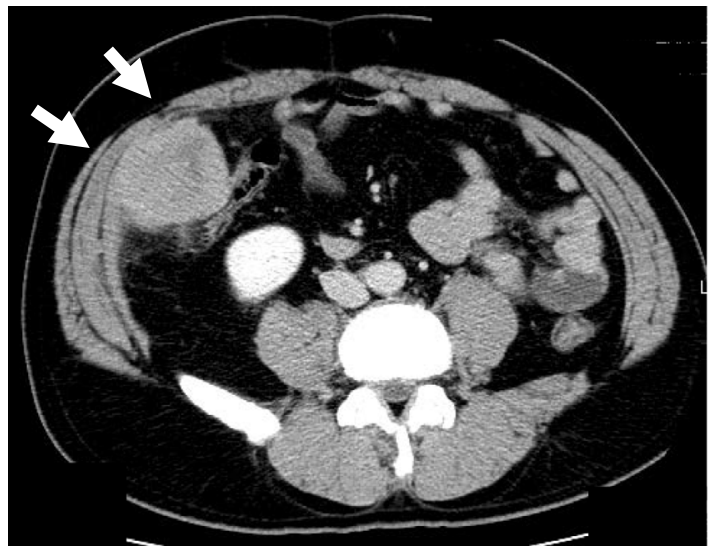

A

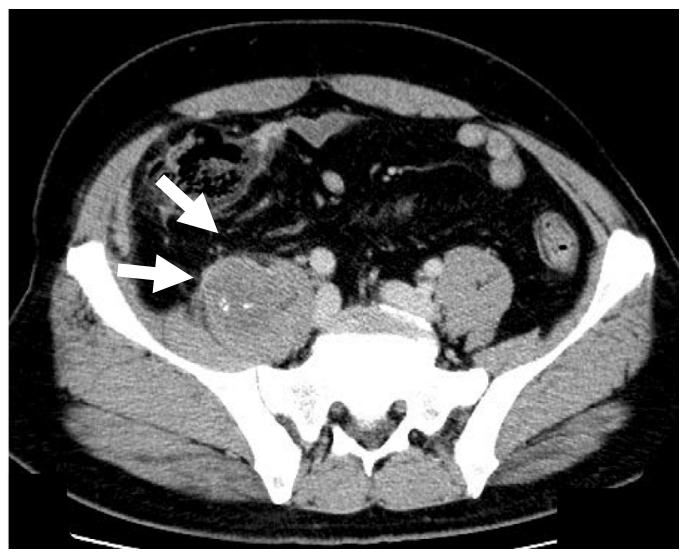

B

Figure 1. Computed tomography (CT) of the abdomen. A: A 7.2 $\times 6.0-\mathrm{cm}$ mass on the right side of the abdomen (white arrows). B: A $3.7 \times 3.6-\mathrm{cm}$ mass in the right iliopsoas muscle (white arrows).

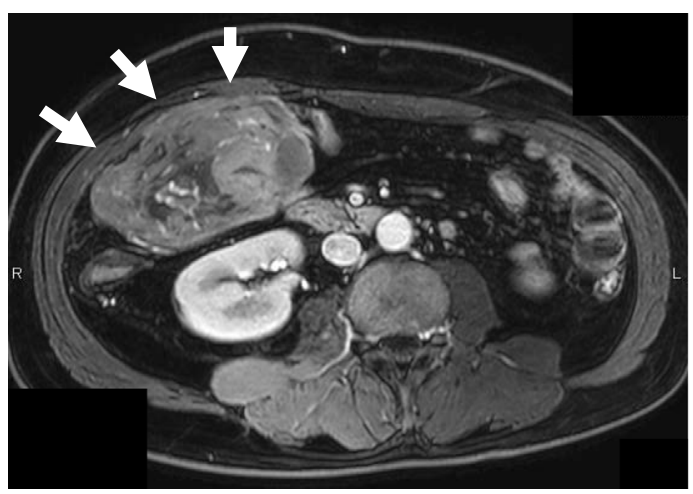

A

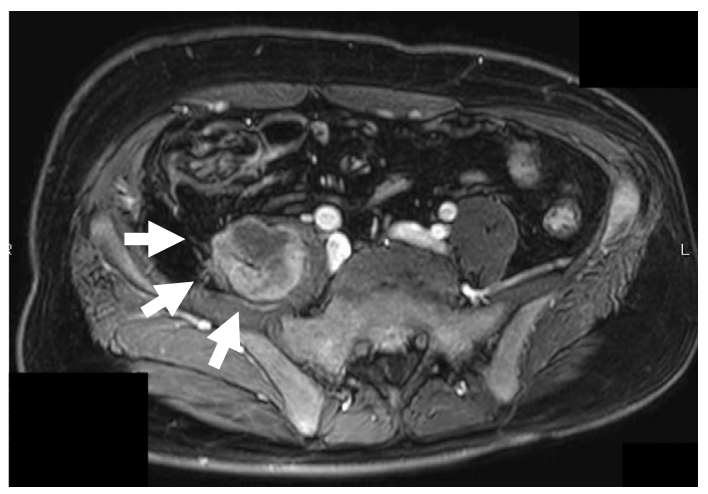

B

Figure 2. Magnetic resonance imaging (MRI) of the abdomen. A: A $12.9 \times 6.8-\mathrm{cm}$ mass on the right side of the abdomen (white arrows) with a high intensity in the early phase, as observed in enhanced images. B: A 3.7×3.6-cm mass in the right iliopsoas muscle (white arrows).

of weight in a month. On a physical examination, his body temperature was $37.4^{\circ} \mathrm{C}$, and a large palpable mass was detected in the right lower abdomen. The hematological examination showed a hemoglobin level of $12.3 \mathrm{~g} / \mathrm{dL}$ (normal range: 14.1-17.2 g/dL), white blood cell count of 21,440/ $\mathrm{mm}^{3}$, and severe elevated serum C-reactive protein (CRP) level of $14.8 \mathrm{mg} / \mathrm{dL}$. The levels of tumor markers, including CEA and CA19-9, were within the normal limits. While a CT-guided biopsy of the masses on the right side of the abdomen and right iliopsoas muscle was performed, the specimens revealed inflammatory changes, mainly composed of neutrophils, so the biopsy was not useful for the diagnosis.

The patient complained of a continuous high fever and was hospitalized for both a further examination and treatment. During hospitalization, his maximum temperature was $40.0^{\circ} \mathrm{C}$ despite antipyretic medication, with a maximum leukocyte count of $40,100 / \mathrm{mm}^{3}(84.1 \%$ neutrophils, $1.2 \%$ eosinophils, $0.1 \%$ basophils, $6 \%$ monocytes, and $8.6 \%$ lymphocytes) and serum CRP level of $21.3 \mathrm{mg} / \mathrm{dL}$. The serum cytokine values were assessed by an enzyme immunoassay, and elevated G-CSF levels of $339 \mathrm{pg} / \mathrm{mL}$ (normal range: 10.5-57.5 pg/mL) and elevated interleukin-6 (IL-6) levels of $110 \mathrm{pg} / \mathrm{mL}$ (normal range: 0-2.41 pg/mL) were noted. Magnetic resonance imaging (MRI) of the abdomen revealed a $12.9 \times 6.8-\mathrm{cm}$ mass on the right side of the abdomen, which showed marked enlargement in 1 month with a relatively homogenous intensity on T2-weighted imaging and high intensity in the early phase on enhanced images accompanied by bleeding caused by the CT-guided biopsy (Fig. 2A). The size of the mass in the right iliopsoas muscle was unchanged, showing the same intensity as that on the right side of the abdomen (Fig. 2B). Colonoscopy revealed only extramural compression in the ascending colon (Fig. 3). ${ }^{18} \mathrm{~F}$ fluorodeoxyglucose-positron emission tomography (FDG$\mathrm{PET} / \mathrm{CT}$ showed the elevated uptake of FDG in the right abdominal tumor [maximum standardized uptake value (SUVmax) 25.12] (Fig. 4A) and tumor in the right iliopsoas muscle (SUVmax 17.84) (Fig. 4B). In addition, an increased FDG uptake in the systemic bone marrow, which indicated elevated bone marrow activity, was noted (Fig. 2B). Al- 
though no obstruction of the large intestine was detected, tumor resection was nevertheless scheduled due to the fact that the patient's high fever persisted.

At the operation, two masses were detected adjacent to the ascending colon invading the right transverse abdominal muscle and right iliopsoas muscle. Right hemi-colectomy with regional lymph node dissection, composite resection of the right transverse abdominal muscle, and resection of the right iliopsoas muscle were performed. No evidence of liver or peritoneal metastases was found.

The high fever and leukocytosis immediately disappeared after resection of the tumors. Serum G-CSF was undetectable after the operation. The postoperative course was un-

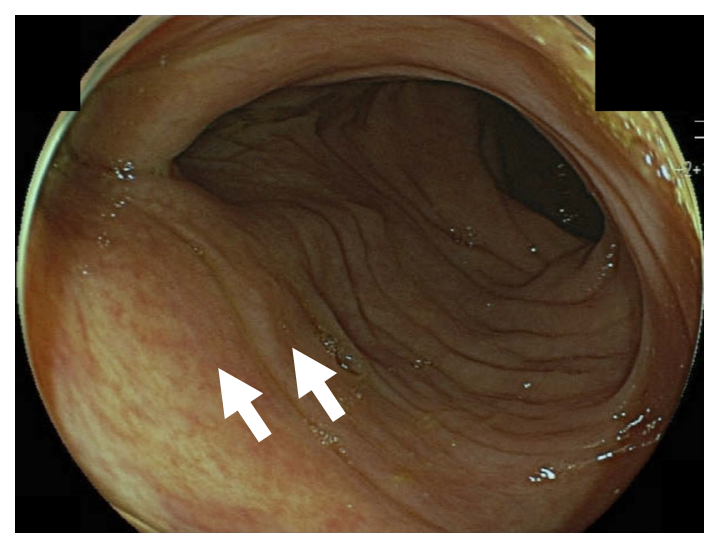

Figure 3. Colonoscopy findings. Extramural compression was revealed in the ascending colon (white arrows).

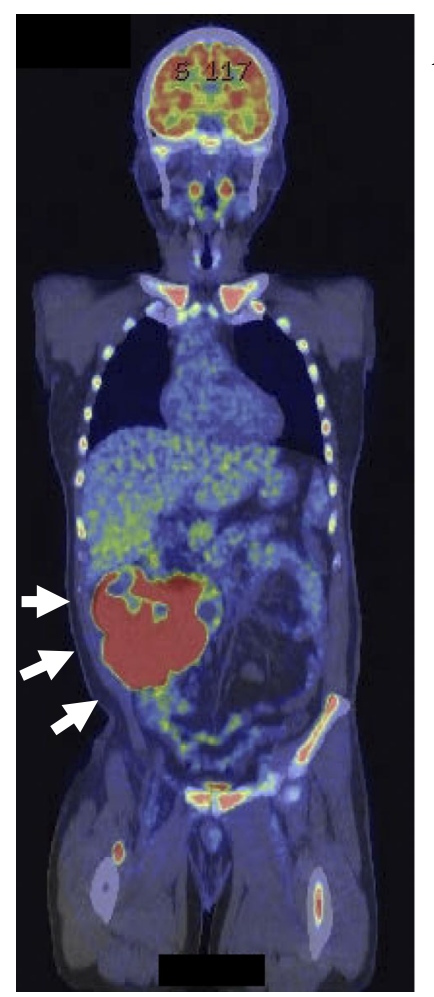

A

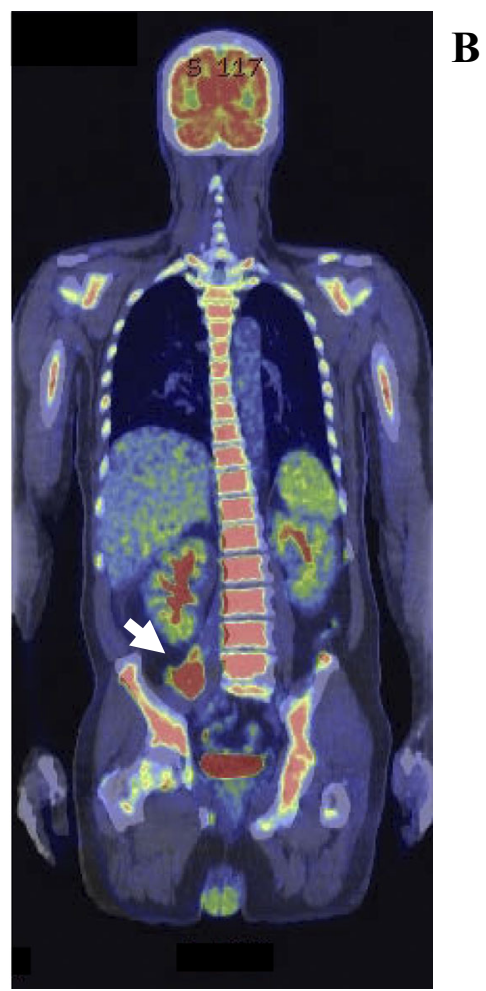

Figure 4. FDG-PET findings before the operation. A: A high uptake of FDG by the tumor in the ascending colon (white arrows). B: A high uptake of FDG by the tumor in the right iliopsoas muscle (white arrow) and a diffuse, low uptake of FDG in the bone marrow. 


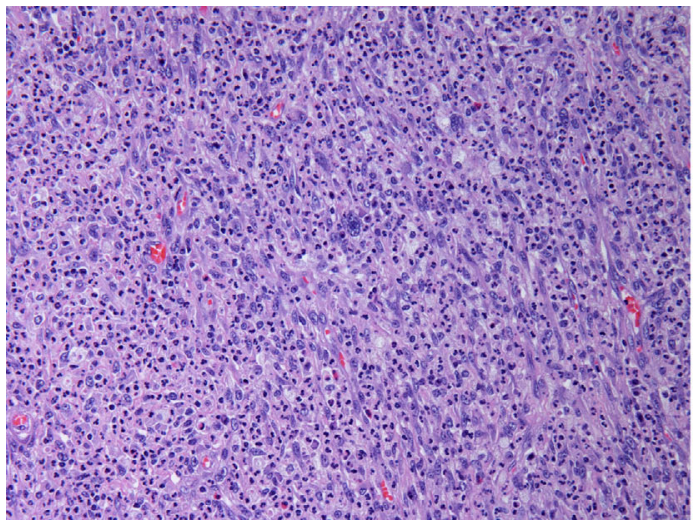

A

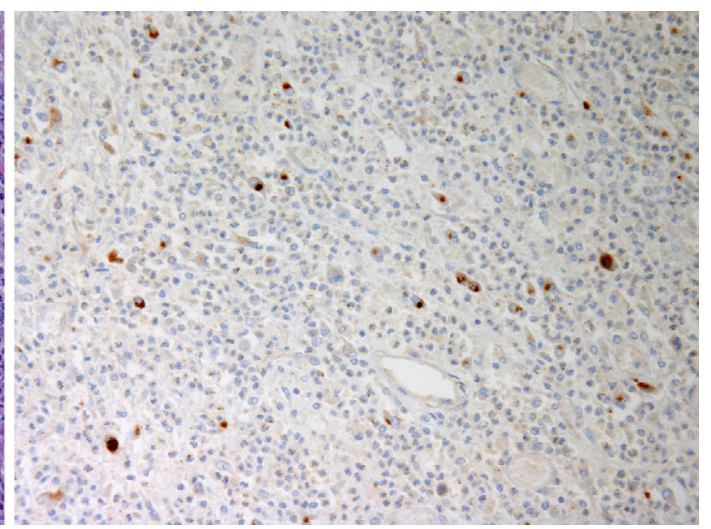

B

Figure 5. Histopathological findings of the resected specimen. A: Fibroblast-like spindle cells with many neutrophils (Hematoxylin and Eosin staining, $\times 200$ ). B: Tumor cells showing positivity for granulocyte colony-stimulating factor $(\times 200)$.

\section{Discussion}

We encountered a case of G-CSF-producing UPS adjacent to the ascending colon and in the right iliopsoas muscle. The patient presented with a high-grade fever, and laboratory tests showed marked leukocytosis and severely elevated serum CRP levels. FDG-PET revealed the localized FDG uptake in both tumors and diffuse uptake in the systemic bone marrow. These inflammatory reactions were considered to have been caused by cytokines, such as serum G-CSF and IL- 6 secreted by tumor cells, and they disappeared immediately after surgical resection.

MFH was first described by O'Brien and Stout in 1964 and it was the most common soft tissue sarcoma found in adults at that time (26). It consists of fibroblastic spindle cells, histiocytic round and polygonal cells, and unusual giant cells. Weiss et al. reported that this tumor most commonly occurred on an extremity (lower 49\%, upper 19\%) and in the abdominal cavity or retroperitoneum (16\%) but rarely in the digestive tract (1). Recently, the classification of soft tissue sarcoma according to the line of differentiation rather than histogenesis has been considered. Therefore, MFH was re-classified as UPS by the World Health Organization in 2002 (23). This tumor did not have any of the typical diagnostic characteristics which could help in performing histological specific differentiation, and therefore an exclusion diagnosis was made by performing an immunohistochemical analysis.

UPS is classified as UPS, UPS with giant cells, and UPS with prominent inflammation.

Primary MFH/UPS of the large bowel is exceedingly rare. We reviewed a total of 23 reported cases of primary colorectal MFH/UPS, including our own case, published in the English literature and summarized the findings in the Table (2-22). The ratio of male to female patients was 19:4, with a median age of 62 years (range 12-80 years). The tumor originated from the cecum in three, ascending colon in seven, transverse colon in four, descending colon in one, sigmoid colon in three, rectum in four, and anal canal in three, including two cases with multicentric tumors. Most of the tumors were large, ranging from 2 to $19 \mathrm{~cm}$ in diameter; their early detection was difficult. All of the patients, excluding one autopsy case, received surgical resection, four of whom received adjuvant chemotherapy, two radiotherapy, and one chemoradiotherapy. Synchronous metastasis was detected in the regional lymph nodes in three patients, the peritoneum in two, and the liver, lymph nodes, and peritoneum in one. Local recurrence was detected in three patients (local alone in one, local and lung in one, and local and liver in one) and lung and liver metastasis in one patient each. Local recurrence was most common in MFH/UPS of the large intestine; the tumors spread radially through the muscle, involving the surrounding structures. Seven cases were considered to be inflammatory MFH/UPS (2, 8, 12, 17, 19, 24).

Recently, inflammatory UPS has been reported to be correlated with the production of G-CSF by tumor cells (12). Furthermore, several studies have reported that G-CSF and IL-6 are produced by lung carcinoma (27). To our knowledge, this is the first study to report that G-CSF and IL-6 are produced by inflammatory UPS. IL-6 is known to play a role in stimulating the production of G-CSF (28). The elevated IL-6 level in previous reports might have contributed to the high-grade fever and elevated serum CRP level (29), and the elevated serum G-CSF level might have contributed to leukocytosis and hematopoietic activation (30). G-CSFproducing tumors are characterized by 1) an increased WBC count, predominantly neutrophils, in the absence of infectious and hematologic diseases; 2) an increased serum GCSF level; 3) normalization of the WBC count and serum G-CSF level after removal of the tumor; and 4) the presence of G-CSF in the tumor tissue (31). In the present case, all of these criteria were fulfilled. FDG-PET imaging of patients with G-CSF-producing tumors has shown not only the elevated uptake of FDG by the primary tumors but also its dif- 


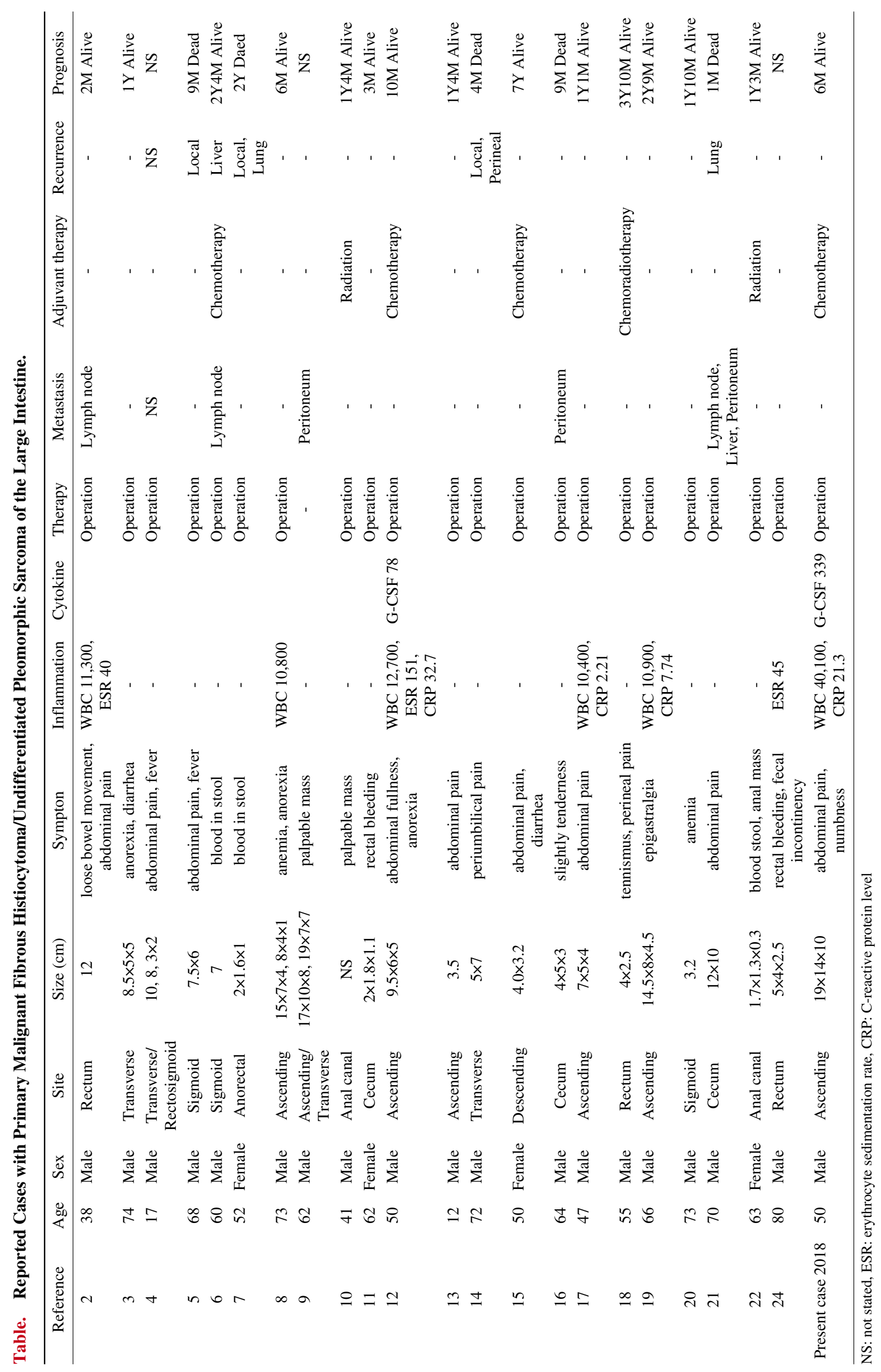


fuse uptake throughout the bone marrow. This FDG finding of bone marrow was thought to indicate that G-CSFproducing tumors enhanced bone marrow metabolism, potentially being useful for the diagnosis of G-CSF-producing tumors (32). In our case, the uptake of FDG was noted in both tumors and the systemic bone marrow, and this signal disappeared after resection of the tumors.

The strategy for treating MFH/UPS is early and complete excision with en-bloc regional lymph node dissection. It has been reported that this tumor in the extremities and limb girdles is not radiosensitive, but radiation therapy plays an important role in achieving local control, particularly in high-grade lesions or in cases with positive surgical margins (33). In the present case, radiation therapy was not performed because the resected margin was negative. Although the role of adjuvant chemotherapy in colorectal MFH/UPS is controversial, 3 patients treated by adjuvant chemotherapy survived for $>10$ months of follow-up $(6,12,15)$. Inflammatory MFH/UPS has been reported to be aggressive with an ultimately fatal course, but four cases with either inoperable or recurrent inflammatory MFH have proven responsive to chemotherapy (34). Therefore, we considered that the present patient should receive adjuvant chemotherapy despite no signs of recurrence for six months after the operation.

In conclusion, although G-CSF-producing colorectal UPS is rare, the clinical condition of leukocytosis (especially of neutrophils), the elevated serum CRP level, and the uptake of FDG in the bone marrow may suggest this tumor type. Changes in these parameters may be useful as markers of tumor recurrence.

The authors state that they have no Conflict of Interest (COI).

\section{References}

1. Weiss SW, Enzinger FM. Malignant fibrous histiocytoma: an analysis of 200 cases. Cancer 41: 2250-2266, 1978.

2. Verma P, Chandra U, Bhatia PS. Malignant histiocytoma of the rectum: report of a case. Dis Colon Rectum 22: 179-182, 1979.

3. Sewell R, Levine BA, Harrison GK, et al. Primary malignant fibrous histiocytoma of the intestine: intussusception of a rare neoplasm. Dis Colon Rectum 23: 198-201, 1980.

4. Levinson MM, Tsang D. Multicentric malignant fibrous histiocytomas of the colon. report of a case and review of the subject. Dis Colon Rectum 25: 327-331, 1982.

5. Waxman M, Faegenburg D, Waxman JS, et al. Malignant fibrous histiocytoma of the colon associated with diverticulitis. Dis Colon Rectum 26: 339-343, 1983.

6. Rubbini M, Marzola A, Spanedda R, et al. Primary malignant fibrous histiocytoma of the sigmoid colon: a case report. Ital J Surg Sci 13: 299-302, 1983.

7. Spagnoli LG, Dell'Isola C, Sportelli G, et al. Primary malignant fibrous histiocytoma of storiform-pleomorphic type: a case report of an ano-rectal localization. Tumori 70: 567-570, 1984.

8. Baratz M, Ostrzega N, Michowitz M, et al. Primary inflammatory malignant fibrous histiocytoma of the colon. Dis Colon Rectum 29: 462-465, 1986.

9. Satake T, Matsuyama M. Cytologic features of ascites in malignant fibrous histiocytoma of the colon. Acta Pathol Jpn 38: 921-
928, 1988.

10. Flood HD, Salman AA. Malignant fibrous histiocytoma of the anal canal. Report of a case and review of the literature. Dis Colon Rectum 32: 256-259, 1989.

11. Katz RN, Waye JD, Batzel EL, et al. Malignant fibrous histiocytoma of the gastrointestinal tract in a patient with neurofibromatosis. Am J Gastroenterol 85: 1527-1530, 1990

12. Murata I, Makiyama K, Miyazaki K, et al. A case of inflammatory malignant fibrous histiocytoma of the colon. Gastroenterol Jpn 28: 554-563, 1993.

13. Huang Z, Wei K. Malignant fibrous histiocytoma of the ascending colon in a child. Am J Gastroenterol 88: 972-973, 1993.

14. Makino M, Kimura O, Kaibara N. Radiation-induced malignant fibrous histiocytoma of the transverse colon: case report and review of the literature. J Gastroenterol 29: 767-771, 1994.

15. Kawashima H, Ikeue S, Takahashi Y, et al. Primary malignant fibrous histiocytoma of the descending colon. Surg Today 27: 851854, 1997.

16. Hiraoka N, Mukai M, Suzuki M, et al. Malignant fibrous histiocytoma of the cecum: report of a case and review of the literature. Pathol Int 47: 718-724, 1997.

17. Udaka T, Suzuki Y, Kimura H, et al. Primary malignant fibrous histiocytoma of the ascending colon: report of a case. Surg Today 29: 160-164, 1999.

18. Singh DR, Aryya NC, Sahi UP, et al. Malignant fibrous histiocytoma of the rectum. Eur J Surg Oncol 25: 447-448, 1999.

19. Okubo H, Ozeki K, Tanaka $\mathrm{T}$, et al. Primary malignant fibrous histiocytoma of the ascending colon: report of a case. Surg Today 35: 323-327, 2005.

20. Bosmans B, de Graaf EJ, Torenbeek R, et al. Malignant fibrous histiocytoma of the sigmoid: a case report and review of the literature. Int J Colorectal Dis 22: 549-552, 2007.

21. Fu DL, Yang F, Maskay A, et al. Primary intestinal malignant fibrous histiocytoma: two case reports. World J Gastroenterol 13: 1299-1302, 2007.

22. Kim BG, Chang IT, Park JS, et al. Transanal excision of a malignant fibrous histiocytoma of anal canal: a case report and literature review. World J Gastroenterol 14: 1459-1462, 2008.

23. Fletcher CD, Unni K, Mertens F. World Health Organization of Tumors: pathology and genetics of tumors of soft tissue and bone. IARC Press, Lyon, 2002: 119-126.

24. Azizi R, Mahjoubi B, Shayanfar N, et al. Malignant fibrous histiocytoma of rectum: report of a case. Int J Surg Case Rep 2: 111113, 2011.

25. Umehara M, Watanabe A, Umehara M, et al. G-CSF producing malignant fibrous histiocytoma in the jejunum: a case report. Hepatogastroenterology 47: 1630-1632, 2000.

26. O'Brien JE, Stout AP. Malignant fibrous xanthomas. Cancer 17: 1445-1455, 1964.

27. Makino T, Hata Y, Otsuka H, et al. Diffuse fluorodeoxyglucosepositron uptake in the bone marrow of a patient with granulocyte colony-stimulating factor-producing pleomorphic carcinoma of the lung: a case report. Mol Clin Oncol 7: 103-106, 2017.

28. Shannon MF, Coles LS, Fielke RK, et al. Three essential promoter elements mediate tumour necrosis factor and interleukin-1 activation of the granulocyte-colony stimulating factor gene. Growth Factors 7: 181-193, 1992.

29. Guo $\mathrm{Y}, \mathrm{Xu} \mathrm{F}, \mathrm{Lu} \mathrm{T}$, et al. Interleukin-6 signaling pathway in targeted therapy for cancer. Cancer Treat Rev 38: 904-910, 2012.

30. Mabuchi S, Morimoto A, Fujita M, et al. G-CSF induces focal intense bone marrow FDG uptake mimicking multiple bone metastases from uterine cervical cancer: a case report and review of the literature. Eur J Gynaecol Oncol 33: 316-317, 2012.

31. Asano S, Urabe A, Okabe T, et al. Demonstration of granulopoietic factor(s) in the plasma of nude mice transplanted with a human lung cancer and in the tumor tissue. Blood 49: 845-852, 1977. 
32. Kuroshima T, Wada M, Sato T, et al. G-CSF producing oral carcinoma with diffuse uptake of FDG in the bone marrow: a case report. Oncol Lett 15: 1241-1245, 2018.

33. Belal A, Kandil A, Allam A, et al. Malignant fibrous histiocytoma: a retrospective study of 109 cases. Am J Clin Oncol 25: 16-22, 2002.

34. Poon MC, Durant JR, Norgard MJ, et al. Inflammatory fibrous histiocytoma: an important variant of malignant fibrous histiocy- toma highly responsive to chemotherapy. Ann Intern Med 97: 858$863,1982$.

The Internal Medicine is an Open Access journal distributed under the Creative Commons Attribution-NonCommercial-NoDerivatives 4.0 International License. To view the details of this license, please visit (https://creativecommons.org/licenses/ by-nc-nd/4.0/).

(C) 2019 The Japanese Society of Internal Medicine Intern Med 58: 2783-2789, 2019 